\title{
Prise en charge de l'arrêt cardiaque : former les citoyens pour... éduquer les citoyens ? Oser AGIR !
}

\author{
Teaching for a resuscitation for cardiac arrest: dare to train citizens to educate citizens!
}

\author{
C. Ammirati \\ (C) SFMU et Springer-Verlag France 2012
}

De nombreux travaux publiés pointent une faible rétention après un enseignement portant sur les gestes d'urgence auprès de la population. Peu d'écrits remettent en cause les stratégies de formation essentiellement fondées sur des méthodes démonstratives par des « instructeurs » spécialistes de la question.

Et si nous changions de paradigme pour diffuser les actions de santé publique en médecine d'urgence dans la population? Si nous portions nos espoirs et nos efforts sur des messages simplifiés scientifiquement justes, sur des méthodes pédagogiques modernes en débutant dès la maternelle, sur la formation et le rôle de « citoyens éducateurs » tout en restant les garants du contenu scientifique?

\section{Construire un savoir depuis le plus jeune âge}

Conformément aux recommandations internationales, depuis 2007, dans le programme « apprendre à porter secours », l'apprentissage du comportement face à une situation d'urgence est obligatoire de la maternelle au collège en France. Le parti pris a été que les « enseignants-citoyens » forment euxmêmes les élèves en fonction de leur développement psychomoteur et en prenant ancrage sur le programme scolaire.

\section{Construire un savoir en apprenant autrement}

Partant des orientations actuelles en sciences de l'éducation, des travaux français relatifs aux apprentissages en santé commencent à être publiés pour montrer l'intérêt de construire un savoir fondé sur les connaissances antérieures (enrichies aujourd'hui par les médias et internet), sur l'essai-erreur et la compréhension plutôt que sur la démonstration. La séquence pédagogique «AGIR : apprendre un geste en intégrant le raisonnement » en médecine d'urgence en est un exemple.

\section{Ammirati $(\square)$}

Pôle anesthésie-réanimation, médecine d'urgence, CHU Amiens, place Victor Pauchet, F-80000 Amiens, France

e-mail : Christine.ammirati@chu-amiens

\section{Construire un savoir par les pairs}

L'apprentissage par les pairs a fait preuve de son efficacité et il est déjà prônée en éducation thérapeutique (" patient éducateur ») mais peu développé dans le domaine de l'urgence.

Le laboratoire en santé de l'université Paris XIII a débuté une action « apprendre à porter secours et soins » avec les associations des familles de France pour que les citoyens se réapproprient les bases simples de l'éducation en santé, y compris en urgence.

En s'appuyant sur ces deux derniers points (méthodes pédagogiques actives et apprentissage par les pairs), le Centre d'enseignement des soins d'urgence (CESU) de SaintEtienne a initié pour des novices une action de formation de «formateurs relais » à la prise en charge de l'arrêt cardiaque en huit heures [1]. La retransmission du savoir théorique et pratique par ces personnes « non expertes » auprès d'autres citoyens semble donner des résultats extrêmement encourageants à terme. Ces résultats sont les prémices d'un changement annoncé : et si nous partions du savoir, du « bon sens » et des représentations de la population ?

Si dans les CESU, dans les universités, nous poursuivions les travaux de recherche en pédagogie notamment sur des modules de formation simplifiés, sur le « oser agir », sur la guidance par téléphone par le médecin régulateur du Samu?

Alors, peut-être que l'action conjuguée de l'apprentissage des gestes d'urgence dès l'école, de la diffusion d'un savoir par les citoyens formateurs relais, de l'obligation actuelle de l'obtention de l'attestation de formation des gestes et soins d'urgence (AFGSU) pour les professionnels de santé, de l'enseignement du secourisme, du libre accès au défibrillateur automatisé externe et de l'aide de la régulation médicale des Samu permettra de renforcer significativement le premier maillon de la chaîne.

\section{Référence}

1. O Durand, H Volle, C Espesson, C Frachon (2012) Les formateurs relais : un dispositif efficace et innovant de formation du grand public à la pris en charge de l'arrêt cardiaque. Ann Fr Med Urg 2:378-83 